\title{
TRIAGEM AUDITIVA: PERFIL SOCIOECONÔMICO DE MÃE
}

\section{Socio-economic characteristic of mothers of a NHS program}

\author{
Maria Luiza Lopes Timóteo de Lima ${ }^{(1)}$, Ana Beatriz Rigueira de Assis ${ }^{(2)}$, \\ Greice Bandeira das Mercês ${ }^{(3)}$, Paulo Freitas de Barros ${ }^{(4)}$, Silvana Maria Sobral Griz ${ }^{(5)}$
}

\section{RESUMO}

Objetivo: descrever as características socioeconômicas e demográficas das mães cujos filhos participaram da triagem auditiva neonatal. Métodos: o estudo foi desenvolvido em maternidades públicas da cidade do Recife, por meio de entrevistas realizadas com as parturientes. Após a entrevista, as mães foram convidadas a comparecer às clínicas-escola de Fonoaudiologia da Universidade Católica de Pernambuco e da Faculdade Integrada do Recife, para realizar avaliação auditiva em seus filhos. Resultados: participaram do estudo 1.021 mães, sendo observado que: a idade destas variou de 13 a 50 anos, com maioria (69,5\%) entre 20 e 35 anos; $59,4 \%$ são casadas ou têm união consensual; $39,1 \%$ são solteiras e a maior parte $(34,1 \%)$ possui primeiro grau incompleto e $3,4 \%$, o $3^{\circ}$ grau. Quanto ao fator renda pessoal, a maioria $(72,8 \%)$ apresenta inferior a um salário mínimo; $90,3 \%$ possuem bens, sendo que $83,6 \%$ televisão, $56,7 \%$ casa própria e $40,7 \%$ telefone e, como atividade, identificou-se $(63,7 \%)$ como sendo dona de casa. Conclusão: as características socioeconômicas e demográficas das mães participantes da triagem auditiva neonatal foram marcadas por mulheres adultas jovens, que possuem marido ou parceiros, baixa escolaridade e renda familiar, possuem acesso à televisão e não possuem trabalho fora de casa. Tais resultados representam uma importante base diagnóstica para as necessárias ações de gestão, assistência e ensino na área de saúde materno-infantil. Portanto, a partir dos achados pode-se melhor fundamentar programas direcionados para as mães com as características socioeconômicas e demográficas identificadas. Entretanto, para se atingir todas as mães, o estudo também forneceu indícios de grupos minoritários que merecem, certamente, novas pesquisas seqüenciais.

DESCRITORES: Fatores Socioeconômicos; Audição; Recém-Nascido

\section{INTRODUÇÃO}

O Brasil é considerado um país em desenvolvimento, por apresentar problemas como: grande desigualdade na distribuição de rendas e difícil acesso e qualidade dos serviços prestados na área

(1) Fonoaudióloga; Professora do curso de Fonoaudiologia Universidade Católica de Pernambuco; Mestre em Saúde Pública pelo Centro de Pesquisas Aggeu Magalhães da Fundação Oswaldo Cruz.

(2) Fonoaudióloga; Especializanda em Audiologia Clínica pela Universidade Católica de Pernambuco.

(3) Fonoaudióloga; Especializanda em Audiologia Clínica pela Faculdade Integrada do Recife.

(4) Fonoaudiólogo; Professor do curso de Fonoaudiologia da Universidade Católica de Pernambuco; Mestre em Fonoaudiologia pela Pontifícia Universidade Católica de São Paulo.

(5) Fonoaudióloga; Professora do curso de Fonoaudiologia da Universidade Federal de Pernambuco; Doutora em Psicologia Cognitiva Universidade Federal de Pernambuco. de educação e saúde ${ }^{1}$. Na conferência de Otawa (1986), foram explicitados os determinantes e as responsabilidades com a saúde a partir de quatro dimensões: 1. As condições, situações e estilos de vida; 2. A situação ambiental; 3. O desenvolvimento da biologia e 4. A organização da assistência à saúde.

Dessa forma, existe convicção de que saúde não é apenas ausência de doença, mas sim boas condições biológicas, ecológicas, ambientais, emocionais, políticas, econômicas, sociais e culturais. Isto depende cada vez mais, de questões políticas, econômicas, sociais e de aspectos culturais, do que do campo da intervenção da medicina propriamente dita. As sensíveis mudanças demográficas, socioeconômicas e culturais também são fatores que interferem de forma influente no que atualmente têm-se como saúde ${ }^{2}$.

No que se refere às deficiências, a Organização Mundial de Saúde (OMS) (1993), estima que 
cerca de $10 \%$ da população brasileira é portadora de alguma delas. Conforme o IBGE (2000), no Brasil existem cerca de 5.750 .809 pessoas portadoras de perda auditiva de grau e etiologia variados, ocupando assim o terceiro lugar entre as deficiências existentes no país.

Levando-se em conta a importância da audição para o desenvolvimento da linguagem, hoje existe um consenso em que se destaca a importância da triagem auditiva em todos os bebês e não mais somente em bebês de alto risco. O Joint Committe on Infant Hearing (2007) entende por triagem auditiva neonatal (TAN) e "intervenção imediata", programas ou um conjunto de ações que envolvam o apoio imediato à família e ao bebê, de preferência, antes dos três meses de idade para o diagnóstico, e antes dos seis meses de idade, para a intervenção ${ }^{3}$.

No entanto, por falta de campanhas oficiais regulares é de se presumir que a sociedade seja desconhecedora de algumas informações da área de saúde auditiva. Esse fato pode ser atribuído a vários fatores, tais como: a falta de informação das mães quanto às causas, os sintomas, os prejuízos de uma perda auditiva no desenvolvimento global da criança; a não valorização da audição como parte dos profissionais da saúde; e a desinformação dos pais sobre a importância dos cuidados gerais com o bebê lactente ${ }^{4,5}$. Esses fatores fazem com que os programas de triagem auditiva neonatal muitas vezes não alcancem os indicadores de qualidade propostos. A evasão encontrada nos programas de triagem auditiva pode ser atribuída, ainda, às condições sócio-econômico-culturais ${ }^{6,7}$.

A educação e os cuidados com a saúde de uma criança deficiente requerem envolvimento de toda a estrutura familiar, gerando custo adicional e diminuição da renda familiar. Portanto, quanto mais cedo for o diagnóstico e o tratamento, melhores e mais rápidos serão os resultados, gerando menos custo e possibilitando a inserção do indivíduo no convívio social ${ }^{8}$.

Considerando que a mãe é a pessoa mais próxima da criança, esta deve reconhecer os benefícios de uma triagem auditiva. Desta forma, o presente trabalho teve por objetivo caracterizar o perfil sócioeconômico e demográfico das mães convidadas a realizar a triagem auditiva neonatal. Faz-se fundamental conhecer este perfil para que os programas de triagem auditiva neonatal sejam voltados de forma específica para as diferentes realidades, com o intuito de melhor sensibilização e acolhimento destas mães.

\section{MÉTODOS}

O estudo foi desenvolvido em cinco maternidades publicas da cidade do Recife. As maternidades estudadas representam as principais referências do município para a assistência à saúde maternoinfantil. O período compreendido para a execução do estudo foi de junho de 2005 a julho de 2006.

Trata-se de uma pesquisa descritiva na qual não houve interferência do pesquisador no objeto de estudo. Procurou-se identificar a freqüência com que os eventos ocorreram, sua natureza, características, causas, relações e conexões com outros fenômenos.

Após o consentimento da instituição, por escrito, o trabalho foi iniciado. As visitas às maternidades ocorrem regularmente, de maneira que todas as mães foram contatadas e convidadas a comparecer às clínicas-escola dos cursos de Fonoaudiologia da Universidade Católica de Pernambuco e da Faculdade Integrada do Recife, para realizar os exames. As visitas às unidades de saúde ocorriam três vezes por semana; assim, todas as mães que estavam internadas para ter o filho ou as que já o tiveram foram contatadas. As que concordaram em participar assinaram o Termo de Consentimento Livre e Esclarecido.

Ainda nas unidades de saúde, as mães foram submetidas a uma entrevista com questões fechadas, que versaram sobre a sua situação socioeconômica e demográfica, gestação e pré-natal e fatores de risco para audição.

Após a entrevista, a avaliação auditiva do bebê foi agendada, pela pesquisadora, nos horários disponíveis para atendimento a esta população, sem nenhum custo para as mesmas. Todos os bebês que estavam em processo de alta da unidade de saúde foram incluídos no estudo, independente de serem prematuros ou portarem síndromes ou comprometimentos neurológicos.

Os bebês que compareceram para realizar o exame auditivo foram submetidos ao teste das emissões otoacústicas, utilizando o equipamento Madsen modelo Capella, no modo Fastscreen, em ambas as orelhas. Após a avaliação, as mães receberam o resultado e as orientações necessárias de acordo com o resultado, os devidos encaminhamentos e um folder explicativo, elaborado pelas pesquisadoras, sobre o desenvolvimento da audição e a importância da avaliação auditiva. Os exames e as orientações foram realizados pelos professores e alunos participantes do estudo. Nos horários de atendimento, cada professor contou com um aluno da graduação e outro da pós-graduação.

Participaram do estudo 1.017 mães, com idades que variaram entre 13 e 50 anos, com média de 
24,50 anos, desvio padrão de 6,30 anos e coeficiente de variação igual a $25,71 \%$. A mediana da idade foi 23 anos.

$\mathrm{Na}$ análise dos dados foram obtidas distribuições absolutas e percentuais para as variáveis nominais apresentadas. Este estudo foi aprovado pelo Comitê de Ética em Pesquisa da UNICAP sob no 076/2004.

\section{RESULTADOS}

Da Tabela 1, destaca-se que a maioria 706 $(69,5 \%)$ das puérperas tinha entre 20 e 35 anos, seguido das que tinham entre 13 e 19 anos 245 $(24,1 \%)$ e apenas $65(6,4 \%)$ tinham mais de 35 anos. Em relação ao estado civil, 604 (59,4\%) mães eram casadas ou tinham união consensual; destas, 398 $(39,1 \%)$ eram solteiras e $15(1,5 \%)$ separadas ou divorciadas. Em relação ao nível de escolaridade, os três maiores percentuais corresponderam às mães com primeiro grau incompleto, primeiro grau completo e segundo grau completo, com valores iguais a $338(34,1 \%), 320(32,3 \%)$ e $272(27,4 \%)$, respectivamente.

A maioria $738(72,8 \%)$ das mães tinha renda pessoal inferior a um salário mínimo, seguido das que tinham renda de um a três $253(25,0 \%)$ salários mínimos e apenas 23 (2,3\%) tinham renda pessoal superior a três salários mínimos. Em relação à renda familiar, observa-se que 633 (67,5\%) recebiam entre um a três salários mínimos e 208 $(22,2 \%)$ possuíam renda familiar inferior a um salário mínimo.

Em relação à ocupação, $629(63,7 \%)$ das mães pesquisadas eram donas de casa, seguido de 83 $(8,4 \%)$ que eram estudantes, $66(6,7 \%)$ empregadas domésticas e de 135 (13,7\%) que tinham outra ocupação distinta das relacionadas (Figura 1).

A Figura 2 mostra que $921(90,3 \%)$ das pesquisadas possuíam bens, sendo que $853(83,6 \%)$ possuíam televisão, $578(56,7 \%)$ possuíam casa própria $415(40,7 \%)$ possuíam telefone e apenas 95 $(9,3 \%)$ possuíam carro.

\section{DISCUSSÃO}

A escolha das variáveis desse estudo trouxe maiores informações sobre a relação de cuidado da mãe para com o filho, fundamentado de forma tal a demonstrar que certas características das mães, como o nível de instrução, renda e estado civil são muitas vezes determinantes para a análise da responsabilidade e da maturidade com a qual a mãe acolhe o filho.

Apesar da maioria das mães estudadas terem idade superior a 20 anos, chama atenção o percen-
Tabela 1 - Distribuição das mães pesquisadas segundo as variáveis socioeconômicas e demográficas

\begin{tabular}{|c|c|c|}
\hline Variável & $\mathbf{n}$ & $\%$ \\
\hline \multicolumn{3}{|l|}{ Faixa etária } \\
\hline 13 a 19 & 245 & 24,1 \\
\hline 20 a 35 & 706 & 69,5 \\
\hline$>35$ & 65 & 6,4 \\
\hline TOTAL $^{(1)}$ & 1016 & 100,0 \\
\hline \multicolumn{3}{|l|}{ Estado civil } \\
\hline Solteira & 398 & 39,1 \\
\hline Casada/União consensual & 604 & 59,4 \\
\hline Divorciada/Separada & 15 & 1,5 \\
\hline TOTAL $^{(2)}$ & 1017 & 100,0 \\
\hline \multicolumn{3}{|l|}{ Grau de escolaridade } \\
\hline Analfabeta & 27 & 2,7 \\
\hline $1^{\circ}$ grau incompleto & 338 & 34,1 \\
\hline $1^{\circ}$ grau completo & 320 & 32,3 \\
\hline $2^{\circ}$ grau completo & 272 & 27,4 \\
\hline $3^{\circ}$ grau & 34 & 3,4 \\
\hline TOTAL $^{(3)}$ & 991 & 100,0 \\
\hline \multicolumn{3}{|l|}{ Renda pessoal } \\
\hline < 1 salário mínimo & 738 & 72,8 \\
\hline De 1 a 3 salários mínimos & 253 & 25,0 \\
\hline > 3 salários mínimos & 23 & 2,3 \\
\hline TOTAL $^{(5)}$ & 1014 & 100,0 \\
\hline \multicolumn{3}{|l|}{ Renda familiar } \\
\hline < 1 salário mínimo & 208 & 22,2 \\
\hline De 1 a 3 salários mínimos & 633 & 67,5 \\
\hline > 3 salários mínimos & 97 & 10,3 \\
\hline TOTAL $^{(6)}$ & 938 & 100,0 \\
\hline
\end{tabular}

(1): Para 19 pesquisadas não se dispõe desta informação.

(2): Para 18 pesquisadas não se dispõe desta informação.

(3): Para 44 pesquisadas não se dispõe desta informação.

(4): Para 97 pesquisadas não se dispõe desta informação.

(5): Para 21 pesquisadas não se dispõe desta informação.

(6): Para 97 pesquisadas não se dispõe desta informação.

tual de mães menores de 19 anos $(24,1 \%)$, uma vez que esta faixa etária abrange a adolescência, a qual corresponde a uma fase biológica e psicologicamente conturbada para o indivíduo ${ }^{9}$. A literatura registra que as adolescentes são um grupo de alto risco obstétrico, quando a mãe possui idade inferior a 13 anos, tem duas vezes e meia a mais possibilidade de gerar um bebê com baixo peso. Tais fatores, prematuridade e baixo peso, são tidos como um dos fatores de risco para as alterações auditivas no RN ${ }^{10,11 .}$.

A gravidez na adolescência tornou-se um problema de saúde pública nesse país. A cada um milhão de adolescentes que engravidam, 84\% não 


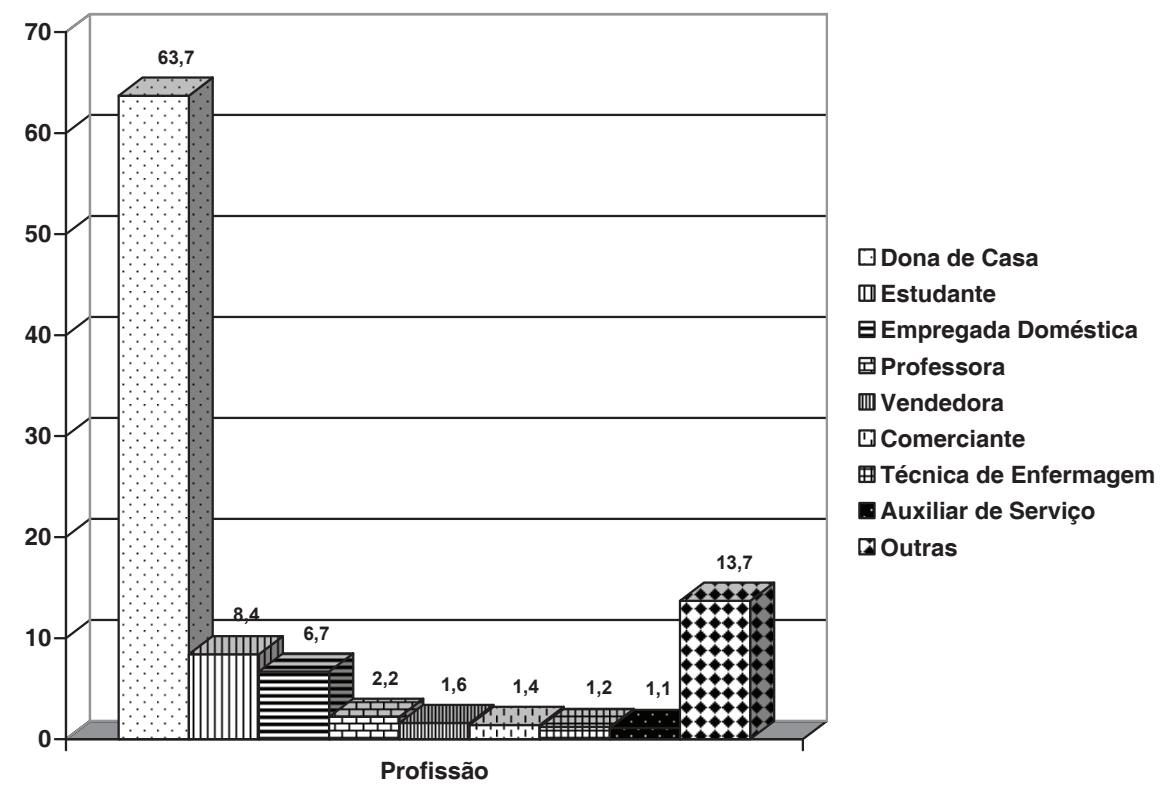

Figura 1 - Distribuição das mães pesquisadas segundo a ocupação

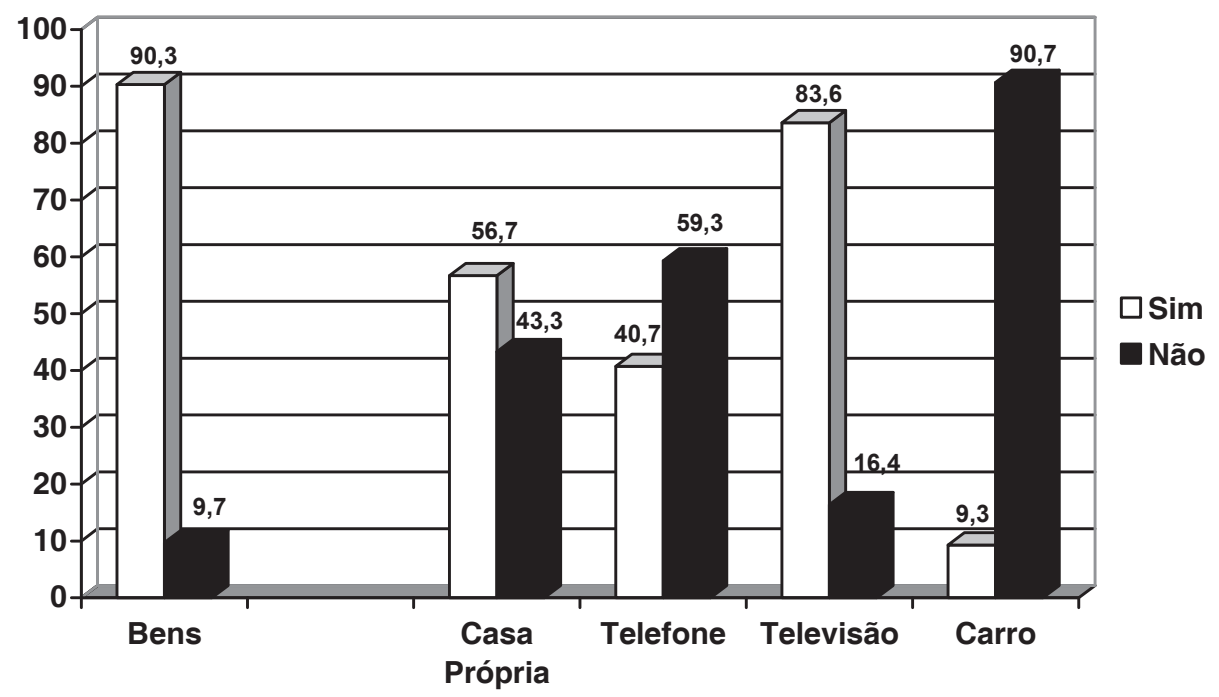

Figura 2 - Distribuição das mães pesquisadas segundo os bens que possuem

querem ser mães, o que favorece o aparecimento de um outro problema, o aborto, algo que também ocorre no Brasil, em todas as classes sociais. $O$ aborto é visto como a solução para o problema da gravidez da jovem adolescente. Porém, muitas vezes, acaba sendo um agravante para condição de nascimento de um feto, que após ser submetido a meios agressivos para interromper sua concepção, quando sobrevivem, podem nascer com malformações diversas ${ }^{12}$, tais como malformações específicas de cabeça e/ou pescoço, consideradas fatores de risco para desenvolvimento de perda auditiva ${ }^{4}$.

No que se refere ao estado civil das mães pesquisadas, identificou-se que em sua maioria $(59,4 \%)$ relatam estar casadas ou possuir união consensual.
Porém, 39,1\% das mães são solteiras. $O$ estado civil é um dado, muitas vezes, significativo, pois o tipo de relacionamento é um dos fatores que motiva a mulher a engravidar e a mãe a se dedicar ao filho. A gestação pode ser vista como a possibilidade de manter uma relação estável com o parceiro, concretizando o sonho do casamento e alcançando autonomia econômica e emocional em relação à família de origem. Viver com o pai do bebê, legal ou consensualmente pode influenciar o modo como a concepção da criança é percebida e aceita pela mãe e sua família ${ }^{12}$.

A existência do apoio conjugal traz reflexos na vivência da gestação, pois o cônjuge é uma das referências mais importantes e que exerce maior 
influência sobre a futura mãe e suas condutas durante e após a gestação ${ }^{13}$. Sendo assim, um dos fatores que melhor predizem a atitude da mãe em relação à gestação é a percepção de que esta é bem aceita pelo pai do bebê ${ }^{14}$. No entanto, as questões sociais também exercem grande influência sobre as condições vitais tanto para a mãe quanto para o bebê ${ }^{15}$.

Considerando que a população do presente estudo apresenta $39,1 \%$ das mães solteiras, mais uma vez atenção deve ser dada a esta população no que se refere a sua conduta durante a gestação, como, por exemplo, auxílio pré-natal e busca de informação sobre o desenvolvimento infantil.

Uma das questões sociais mais importantes é a evasão escolar. A escola possui importância fundamental na educação de um indivíduo. Normalmente, serve como uma continuação ou complementação da educação recebida no âmbito familiar, possibilitando conhecimentos não apenas acadêmicos, como também orientações quanto ao próprio desenvolvimento do jovem ${ }^{16}$. A falta de escolaridade completa, associada à gestação precoce, pode trazer graves conseqüências para a gestante, seu filho e para a sociedade em geral, uma vez que o nível de instrução é visto como um fator social que pode exercer certo controle sobre a vida sexual das mulheres e, conseqüentemente, sobre as chances de gravidez ${ }^{17}$.

Em países em desenvolvimento, uma das poucas opções de inserção social e de ascensão econômica ocorre por intermédio do sistema educacional ${ }^{7}$. Se acrescido a essa discussão, identifica-se que a ocorrência de gestação durante a adolescência é maior entre as camadas menos favorecidas, aumentam-se as chances de gestações de risco, com possíveis conseqüências no desenvolvimento do sistema auditivo de um feto.

Em relação ao nível educacional, o estudo, mais do que o trabalho é um fator determinante na melhoria das condições de vida das pessoas, definindo suas perspectivas futuras e, por isso, a importância do fator escolaridade na vida das adolescentes.

Estudos ${ }^{18,19}$ demonstraram que $80 \%$ dos partos de gestantes são realizados pelo SUS. Esse dado vem a reforçar que as famílias possuem, em sua maioria, renda familiar limitada. Essa condição agrava o risco não só da mãe como da criança, somando-se a isso os diversos problemas sociais e econômicos que essas mães têm de enfrentar, o risco se torna ainda maior.

A distribuição das mães segundo a renda pessoal e familiar é caracterizada, pela ocorrência em sua maioria $(72,8 \%)$ de mães que tem renda pessoal menor que um salário mínimo e $67,5 \%$ que têm sua renda familiar entre um e três salários mínimos. Como começar a trabalhar se torna uma tarefa quase impossível diante de uma gravidez, os fatores econômicos se associam à desistência dos estudos ${ }^{18}$.

A rede de complexidade observada pode levar a característica da população no que se refere à profissão das mães. São 63,7\% as mães que relataram ser donas de casa, implicando alta ocorrência de renda pessoal menor que um salário mínimo. Além disso, muitas não têm renda pessoal. Diante de tal realidade, estas mães tornam-se mais dependentes financeiramente, seja da família, seja do companheiro. Paralelamente, a tendência para estabelecer uniões leva a uma situação sócio-econômica ainda mais precária, o que pode torná-las mais expostas a outras situações de risco social ${ }^{15}$.

Associado a renda pessoal e familiar, identifica-se que o acesso a bens como casa própria, telefone, televisão e carro, está disponível para as mães entrevistadas.

As condições de moradia são fatores relevantes, pois destas, dependerão as condições de alimentação, saúde e higiene (como, acesso a saneamento básico) a que essas mães e crianças, muitas vezes, são submetidas, em uma fase tão frágil de suas vidas ${ }^{19}$. Destaca-se que o acesso à televisão (mais de $80 \%$ das famílias pesquisadas) pode ser um fator positivo devido ao grande poder de alcance e forte instrumental para as ações de educação em saúde.

Atualmente, acredita-se que os riscos presentes durante a gravidez são bastante determinados por fatores psicossociais relacionados ao ciclo da pobreza, à falta de educação formal existente, e fundamentalmente, à falta de perspectivas na vida dessas mães sem escola, saúde, cultura, lazer e emprego ${ }^{11}$.

Considera-se a caracterização destas mães como essencial para que se possa definir que tipo de programa de saúde auditiva a ser implementado, especificamente programas de triagem auditiva neonatal. A partir da compreensão sócio-econômica e demográfica das famílias a serem beneficiadas por um programa, entende-se o vínculo estabelecido com seus filhos e, posteriormente, desenvolver programas de saúde mais eficazes.

Este estudo sugere que haja estruturação de programas de triagem auditiva neonatal, baseada em tais fatores, ou a re-estruturação daqueles realizados rotineiramente nos serviços públicos de assistência à saúde. Os serviços públicos necessitam de políticas e programas eficientes, voltados especificamente para o atendimento de mães com as características descritas nesta pesquisa, contribuindo na estruturação dos programas de saúde auditiva no Brasil, especialmente do Nordeste. 


\section{CONCLUSÃO}

Com o estudo identificou-se que as principais características socioeconômicas e demográficas das mães foram: idade superior a 20 anos de idade, porém com percentual importante de mães adolescentes; mais da metade delas com algum vínculo de união formal ou consensual com seu companheiro; escolaridade predominante o primeiro grau incompleto.

A renda pessoal das mães foi registrada como ausente ou muito baixa, o mesmo se aplica à renda familiar, com registro de pequeno aumento. As informações confirmadas com o registro de que a maioria das mães está desempregada ou são donas de casa. Destaca-se o grande acesso das mães ao meio de comunicação oferecido pela televisão, podendo ser este importante instrumental para os programas de triagem auditiva.

Por fim menciona-se a importância de que a mãe tenha o mínimo de esclarecimento, renda mensal ou condições financeiras, e harmonia no convívio social e com o cônjuge para buscar para o seu filho as melhores ações de saúde.

\section{AGRADECIMENTOS}

Agradecemos especialmente a cada mãe e bebê que se colocaram como voluntárias; as unidades de saúde que disponibilizaram os seus espaços; as instituições de ensino, UNICAP, UFPE e FIR pelo apoio técnico e ao CNPq pelo apoio financeiro disponibilizado.

\section{ABSTRACT}

Purpose: to describe the socio-economic and demographic aspects of mothers related to a newborn hearing screening program in Recife. Methods: this study was developed in public hospitals in Recife, through interviews carried out with the mothers. After each interview, the mothers were invited to carry out hearing screening in their babies in audiological clinics at the Universities, with no any burden. Results: 1,021 mothers took part in this study, with the following features: age varying from 13 to 50 years, with the majority $(69.5 \%)$ between 20 and 35 years-old, $59.4 \%$ were married or had formal consented union, $39.1 \%$ were single, and the majority of mothers (34.1\%) did not finished high school, $3.4 \%$ completed university graduation, the majority of the mothers $(72.8 \%)$ earned less than one minimal wage per month, $90.3 \%$ had goods such as TV $(83.6 \%)$, their self-owned house $(56.7 \%)$, and telephone (40.7\%). Conclusion: the results showed the socio-economic and demographic aspects of the mother involved in the newborn hearing screening program were married young adults, with low educational level and low income. They mostly had no outside job and owned a TV set. Therefore, from de findings we can better substantiate programs direct towards mothers with the indicated socioeconomic features. However, for reaching all mothers, the study also provided indications of minority groups that deserve, certainly, new sequential studies.

KEYWORDS: Socioeconomic Factors; Hearing; Infant, Newborn

\section{REFERÊNCIAS}

1. Henriques R, organizador. Pobreza e desigualdade no Brasil. Rio de Janeiro: IPEA; 2001.

2. Minayo MCS. Tendências do campo da saúde no Brasil no século XXI. In: Bricenõ R, Minayo MCS, Coimbra CE, organizadores. Ciência e tecnologia para século XXI. Rio de Janeiro: Academia Brasileira de Ciências; 2002. p. 46-79.

3. Yoshinaga-Itano C. Levels of evidence: universal newborn hearing screening (UNHS) and early hearing detection and intervention systems (EHDI). J Commun Disord. 2004 sep/oct; 37(5):451-65.
4. Joint Committee on Infant Hearing. Year 2007 Position statement: principles and guidelines for early hearing detection and intervention programs. Pediatrics. 2007; 120(4):898-921.

5. Oliveira TMT, Zanelli AC, Mainardi J. Conhecimento e atitudes das mães frente à triagem auditiva neonatal universal. Fonoaudiol Brasil. 1998; 1:18-21.

6. Olusanya BO, Okolo AA. Early hearing detection at immunization clinics in developing countries. Int $\mathrm{J}$ Pediatr Otorhinolaryngol. 2006; 70(8):1495-8.

7. Davis A, Hind S. The newborn hearing screening programmeinEngland. IntJPediatrOtorhinolaryngol. 2003; 67(S1):193-6. 
8. Martines F, Porrello M, Ferrara M, Martines M, Martines E. Newborn hearing screening project using transient evoked otoacoustic emissions: Western Sicily experience. Int J Pediatric Otorhinolaryngol. 2007 jan; 71(1):107-12.

9. Shoup AG, Owen KE, Jackson G, Laptook A. The Parkland Memorial Hospital experience in ensuring compliance with universal newborn hearing screening follow-up. J Pediatr. 2005 jan; 146(1):66-72.

10. Martinez OS, Bonal AF, Bonal AF, Valido JS, Temes Junior RM. Embarazo en la adolescencia y factores sociodemográficos en Guantánamo en 1998. Rev Cubana Med Gen Integr. 2004; 20(1):80-9.

11. Mioto RCT. A maternidade na adolescência e a (des)proteção social. Serviço Social e Sociedade. 2005; 26(83):128-46.

12. Calvasina PG, Nations MK, Jorge MSB, Sampaio HAC. "Fraqueza de nascença": sentidos e significados culturais de impressões maternas na saúde infantil no nordeste brasileiro. Cad Saud Public. 2007 fev; 23(2):371-80.

13. Stern C. El embarazo en la adolescencia como problema público: una visión crítica. Salud Pública Mex. 1997 mar/abr; 39(2):137-43.
14. Cowley C, Farley T. Adolescent girls' attitudes toward pregnancy: the importance of asking what the boyfriend wants. J Fam Pract. 2001; 50:603-7. 15. Sabroza AR, Leal MC, Gama SGN, Costa JV. Perfil sócio-demográfico e psicossocial de puérperas adolescentes do Município do Rio de Janeiro, Brasil - 1999-2001. Cad Saud Public. 2004; 20(1): S112-20.

16. Chalem E, Mitsuhiro SS, Ferri CP, Barros MCM, GuinsburgR, LaranjeiraR. Gravideznaadolescência: perfil sócio-demográfico e comportamental de uma população da periferia de São Paulo, Brasil. Cad Saúde Publ. 2007 jan; 23(1):177-86.

17. Linares JR, Romero GE, Moreno H. Factores de riesgo de salud maternoinfantil em madres adolescentes de Colômbia. Rev Panam Salud Publica. 1998; 4(2):80-6.

18. Yaslle MEHD, Mendes MC, Patta MC, Rocha JSY, Azevedo GD, Marcolin AC. A adolescente grávida: alguns indicadores sociais. Rev Bras Ginecol Obstetríc. 2002 24(9):609-14.

19. Simões VMF, Silva AAM, Bettiol H, Lamy-Filho F, Tonial SR, Mochel EG. Características da gravidez na adolescência em São Luiz, Maranhão. Rev Saud Publ. 2003; 37(5):559-65.

RECEBIDO EM: 24/09/2007

ACEITO EM: 09/04/2008

Endereço para correspondência:

Rua José Bonifácio, 125 ap. 502

Recife - PE

CEP: $50710-000$

Tel: (81) 3226-0505 / 9914-5968

Fax: (81) 3227-9901

E-mail: mluiza@unicap.br 